\title{
LA CONSERVATION DU SANG
}

\author{
M. Boulanger, B. Paiement, et J.G. Maillé
}

\section{INTRODUCTION}

UNE ENQUÊTE-MAISON toute simple nous a révélé qu'un seul sur 10 médecins interrogés, avait donné de son sang durant l’année écoulée; on a retrouvé la même proportion parmi l'auditoire du dernier symposium annuel de la Société Canadienne des Anesthésistes. Cette proportion supporte mal la comparaison avec celle de la population en général où l'on estime à partir des données de la Croix Rouge, ${ }^{1}$ qu'une personne sur 20 fait un don de sang annuellement. D'autre part, nous médecins, sommes d'ardents consommateurs du sang de nos frères, comme l'illustre le tableau I.

En 1973, sur un million de flacons de sang recueillis par la Croix Rouge, 900,000 ont été fournis aux hôpitaux; plus de 200,000 de ces flacons furent retournés périmés, faute d'utilisation dans les délais prescrits. Un tel pourcentage de stase ( 24 pour cent) n'est pas sans inquiéter les autorités qui y voient un gaspillage d'énergie et d'argent. Soulignons de plus que 5.5 pour cent de tous les flacons receuillis se perdent annuellement: c'est-à-dire non récupérables en constituant du sang.

Force nous est donc d'avouer que nous n'utilisons peut-être pas les ressources actuelles de façon rationnelle. D'autre part, certains indices laissent prévoir une diminution du nombre de donneurs; en effet, on constate une certaine désaffection de la jeunesse actuelle à l'égard du don de sang et de plus, le dépistage des donneurs positifs à l'antigène Australien se faisant de plus en plus complet, éliminera un nombre croissant de donneurs éventuels.

Le tableau II nous montre l'incidence de positivité à l'antigène Australien par 100,000 de population. ${ }^{2}$ A l'analyse, une telle incidence de positivité parmi les donneurs représente le rejet de quelques 1500 flacons annuellement, ce qui est relativement peu, cependant, on estime que le nombre de porteurs positifs est appelé à s'accroître; en effet, plusieurs facteurs contribuent à la diffusion de l'hépatite $\mathrm{B}$ ( tableau III). Il est évident que l’on arrivera jamais à éliminer complètement tous ces facteurs. Quant aux besoins futurs, ils iront sans aucun doute en augmentant: on pratique de plus en plus d'opérations chirurgicales, de nouvelles opérations apparaissent. Roche et Stengle ${ }^{3}$ prévoyaient en 1972 une augmentation exponentielle du nombre d'opérations pour pontages aorto-coronariens et ils prédisaient pour 1976, la possibilité de voir un demi million de ces pontages effectués chaque année. Il semble que la prédiction soit en vo:e de se réaliser. ${ }^{4}$ Ainsi devant

Département d'Anesthésiologie et de Réanimation, Université de Montréal et Institut de Cardiologie de Montréal.

Ce travail a été présenté, en anglais, lors du Symposium "The Clinical Aspects of Blood Transfusion," tenu sous l'égide de la Sociêté Canadienne des Anesthésistes lors de son congrès annuel d St-Jean, Terre-Neuve, le 17 juin 1974. 
TABLEAU I

SANg: Ressources de la Croix Rouge (1973)

\begin{tabular}{lr}
\hline Flacons recueillis & 973,986 \\
Flacons livrés aux hôpitaux & 898,095 \\
Flacons périmés (retournés) & 218,787 \\
Flacons perdus & $55.4 \%)$ \\
\hline
\end{tabular}

TABLEAU II

Antigk̀ne Australien: Porteurs PAR 100,000/POP.

\begin{tabular}{llll}
\hline Toronto & 429 & & \\
Montréal & 481 & Hommes & 5 \\
Canada & 144 & Femme & 1 \\
\hline
\end{tabular}

TABLEAU III

Hepatite B: Facteurs EPIDEMIOLOGIQUES

\begin{tabular}{l} 
Médicaments immuno suppresseurs \\
Transfusions sanguines \\
Emploi non-médical des drogues \\
Contacts vénériens \\
Tatouage (Moustiques) \\
Grossesse (Mère à enfant) \\
Manipulations d'échantillons de sang \\
\hline
\end{tabular}

des ressources limitées et une demande pratiquement illimitée, il nous faut, nous médecins, adopter des attitudes et employer des techniques qui nous permettront de sauver du sang. Ce travail traitera de ces attitudes et de ces techniques.

\section{Attitudes et motivation}

Tout effort en ce sens doit commencer par l'éducation à tous les niveaux d'enseignement. Dans un contexte facultaire, on imagine facilement le département d'anesthésie-réanimation exercer un certain leadership dans l'enseignement de l'utilisation rationnelle du sang et de ses dérivés; après tout, l'anesthésisteréanimateur est probablement de tous les médecins, celui qui est appelé le plus souvent à en poser l'indication.

Au niveau pré-doctoral, il serait intéressant d'analyser ce qu'on enseigne dans nos différentes écoles de médecine; il est à espérer que les connaissances de nos diplômés sur ce sujet dépassent celles contenues dans un bon article de vulgarisation type Readers' Digest ou Ladies Home Journal.

Au niveau post-doctoral, les programmes de formation en anesthésiologie, chirurgie, gynéco-obstétrique, hématologie et médecine intensive, doivent contenir de toute nécessité des périodes d'enseignement spécifiquement consacrées à l'utilisation du sang et de ses dérivés. De tels programmes devraient inclure:

- connaissances d'usage des mécanismes de coagulation,

- connaissances d'usage des coagulopathies importantes,

- indication d'une transfusion de sang et de ses dérivés,

- complications des transfusions, massives ou non,

- méthodes de conservation du sang. 
Au plan de l'éducation médicale continue et en cours de carrière, les anesthésistes se doivent de collaborer aux travaux des comités de la Banque de Sang de leur milieu. Ces activités sont très rentables si l'on considère que l'implantation dans un milieu d'un comité d'étude des transfusions réduit habituellement le nombre de celles-ci de 20 pour cent. ${ }^{5}$

Les avantages strictement quantitatifs d'attitudes judicieuses dans l'emploi du sang sont énormes si l'on s'arrête à considérer avec Garot Allen ${ }^{6}$ que "l'épargne d'un seul flacon par épisode transfusionnel, permettrait de réduire la consommation globale de sang de 40 pour cent."

Un tel objectif, pour être probablement irréalisable intégralement, n'en démontre pas moins la nécessité d'exercer une saine vigilance.

Autre exemple des bienfaits de la vigilance, une étude de l'épidémiologie des transfusions uniques (single unit transfusion) par Reece et Beckett ${ }^{7}$ a montré que le tiers seulement de celles-ci était justifié. Cependant, à ce sujet, il faut se garder d'attitudes inflexibles car la transfusion d'un seul flacon peut être défendable là où elle a contrindiqué l'emploi de deux flacons. ${ }^{6, \tau}$

En plus d'exercer une bienfaisante vigilance, les comités d'étude des transfusions maintiennent un système de dépistage des hépatites post-tranfusionnelles.

Des informations pertinentes diffusées par un bulletin périodique hospitalier émanant de la banque de sang contribuent grandement à l'efficacité des comités d'étude des transfusions. ${ }^{8}$

$\mathrm{Au}$ plan des relations publiques, il y aurait lieu de reviser certaines de nos positions. On a trop longtemps compté sur la Croix Rouge et ses relations publiques pour assurer la motivation du public à l'égard du don de sang. Une récente communication des services de transfusions de la Croix Rouge du Québec incitait les médecins à convaincre les amis et les parents des malades à donner de leur sang; la réponse des familles à ce genre de renquète est habituellement très généreuse: on donne plus facilement son sang par amour pour son proche parent que par vague devoir civique. Nous avons là nous médecins, un rôle de premier plan à jouer.

Une autre position mérite d'être revisée: cette vieille pratique traditionnelle de préparer du sang pour des types d'opérations où on n'en emploie pratiquement jamais. Une analyse portant sur une période de 10 ans dans un grand hôpital a révélé que seulement 3.6 pour cent de tout le sang préparé pour cholécystectomie était effectivement utilisé. ${ }^{9}$ Ceci représente un fardeau inutilement assumé.

Shier ${ }^{10}$ propose à chaque malade prévu pour chirurgie élective de donner préalablement un flacon de son propre sang qui lui sera remis si nécessaire durant la chirurgie; si non employé, ce sang pourvoie la banque de sang pour d'autres besoins.

\section{Techniques de conservation du sang}

Avant d'étudier les techniques elles-mêmes, soit: l'hypotension contrôlée, l'hémodilution et la récupération du sang perdu, un mot sur la prévention des pertes sanguines. Le tableau IV énumère quelques situations où la prémonition, la préparation et la vigilance contribueront toutes à la conservation du sang. 


\begin{tabular}{ll}
\hline \hline Prémonition & $\begin{array}{l}\text { ecchymoses au moindre choc } \\
\text { saignement excessif après extraction dentaire } \\
\text { anticoagulothérapie (acide acétylsalycilique) } \\
\text { insuffisance hépatique }\end{array}$ \\
Préparation & $\begin{array}{l}\text { Porteurs connus de troubles de la coagulation } \\
\text { Vigilance }\end{array}$ \\
& $\begin{array}{l}\text { Eviter rétention de } \mathrm{CO}_{2} \\
\text { congestion veineuse } \\
\text { mauvaise technique chirurgicale }\end{array}$ \\
\hline
\end{tabular}

TABLEAU $V$

Hypotension Contrôlée Pertes Sanguines

\begin{tabular}{lccrc}
\hline \hline & \# cas & $\begin{array}{c}\text { Hypotension } \\
\text { contrôlée }\end{array}$ & \# cas & Contrôle \\
\hline Dissection radicale (aine) & 3 & 295 & 5 & 2080 \\
Anastomose porto-cave & 5 & 890 & 42 & 4280 \\
Crâniotomie & 5 & 520 & 5 & 910 \\
Anévrysme cérébral & 3 & 0 & $\overline{7}$ & $\overline{770}$ \\
Tumeur naso-pharynx & 2 & 45 & $-\frac{7}{7}$ & $\mathbf{1}$ \\
Rhinoplastie & 3 & 75 & 2 & 1415 \\
Dissection radicale (cou) & 6 & 545 &
\end{tabular}

Eckenhoff, et al. Ann. N.Y. Acad. Sciences 115-341 (1964).

\section{TABLEAU VI}

Hypotension Contrólée

\begin{tabular}{ll}
\hline 1942 & Anesthésie profonde \\
1946 & Artériotomie (Gardner) \\
1948 & Blocage sous-arachnoïdien (Gillies and Griffith) \\
1951 & Bloqueurs ganglionnaires (Enderby) \\
1955 & Vasodilatateurs Nitroprussiate de Na (Page, Enderby) \\
1960 & Halothane (Murtagh) \\
\hline
\end{tabular}

\section{Hypotension contrólée}

Cette technique quelque peu audacieuse fut d'abord reçue avec enthousiasme mais de tragiques complications n'ont pas tardé à survenir et ont inspiré à Little en 1956, une monographie ${ }^{11}$ qui a contribué à refroidir cet enthousiasme. Cependant, la technique a survécu et s'emploie encore aujourd'hui en chirurgie neurologique, orthopédique, plastique et en chirurgie du cancer. La méthode permet-elle d'épargner du sang? Sans aucune espèce de doute comme l'indique clairement le tableau V. ${ }^{12}$

Le tableau VI relate l'évolution historique de l'hypotension contrôlée..$^{13}$ Comme adjuvant à ces différentes techniques, ajoutons qu'on utilise habituellement des modifications de la position du malade (ce qui représente de l'hypotension orthostatique) et l'élévation de la pression moyenne intra-thoracique.

Ainsi, il faut bien comprendre que l'hypotension contrôlée est une méthode comportant plusieurs accrocs à la physiologie normale.

De nos jours, les agents les plus communément employés pour réaliser l'hypotension contrôlée sont du type bloqueurs ganglionnaires, tels le trimetaphan ou 
du type vasodilateur périphérique, tel le nitroprussiate de soude. Ce travail n'a pas lintention de revoir la pharmacologie des agents hypotenseurs mais les points suivants méritent d'être soulignés: Jordan et coll. ${ }^{14}$ ont démontré que le trimétaphan possède une action dépressive sur le myocarde qui se compare en intensité à celle de l'halothane; cette constatation est plutôt inattendue. Ainsi à ce jour, on a montré que toutes les méthodes employées pour baisser la tension artérielle, s'accompagnaient du même coup d'une réduction du débit cardiaque.

Cependant d'emploi plus récent, le nitroprussiate de soude semble très prometteur; des communications récentes font état de son emploi pour l'anesthésie hypotensive et pour la thérapeutique des crises hypertensives et du choc cardiogénique. ${ }^{16-10}$

Tout ceci représente de nouvelles indications pour un vieux médicament: en effet, on connaît cet agent depuis plus de 75 ans, mais on a d'abord pensé que sa toxicité était due à la formation de cyanure à la suite de son administration. En 1929, on découvre que la toxicité du médicament est attribuable à son effet hypotenseur.

Boxer et Rickards ${ }^{20}$ étudiant le thiocyanate et les cyanures marqués au carbone ont trouvé que ces deux produits étaient en équilibre dynamique, la réaction au point d'équilibre étant fortement en faveur du thiocyanate. De même Goldstein²1 a montré que des niveaux de thiocyanate aussi élevés que $700 \mathrm{mg}$ pour cent $\mathrm{ml}$ de sang entraînaient une concentration sanguine de cyanure minime se situant entre 10 et $50 \mathrm{mcg}$ pour cent $\mathrm{ml}$ de sang. La plus basse cyanurémie capable d'entraîner un empoisonnement mortel se situerait autour de $340 \mathrm{mcg}$ pour cent ml de sang.

Des travaux récemment publiés ${ }^{22}$ supposent que le nitroprussiate de soude abaisse la tension artérielle sans diminuer le débit aux tissus assurant ainsi un transport d'oxygène suffisant.

Franciosa et ses collaborateurs ${ }^{23}$ ont démontré une nette amélioration de la fonction ventriculaire par l'infusion de nitroprussiate de soude chez des infarcisés du myocarde. Chez 15 de ces malades, l'infusion de nitroprussiate a permis de diminuer la pression ventriculaire télédiastolique et augmenter le débit cardiaque sans modification du travail du cœur. Le médicament semble diminuer la précharge par dilatation veineuse et la postcharge par la diminution de la résistance artériolaire.

Le tableau VII compare les effets et les modes d'action de trois médicaments hypotenseurs couramment employés.

TABLEAU VII

Agents Hypotenseurs

\begin{tabular}{lccc}
\hline & Trimetaphan & $\begin{array}{c}\text { Nitroprussiate } \\
\text { de Na }\end{array}$ & Halothane \\
\hline Vasodilatation & $\begin{array}{c}\text { par blocage } \\
\text { ganglionnaire }\end{array}$ & Directe & $\begin{array}{c}\text { Directe et } \\
\text { centrale }\end{array}$ \\
Débit cardiaque & $\downarrow$ & 0 & $\downarrow$ \\
Pression artérielle & $\downarrow$ & $\downarrow$ & $\downarrow$ \\
Flot sanguin & $\downarrow$ & 0 & $\downarrow$ \\
\hline
\end{tabular}




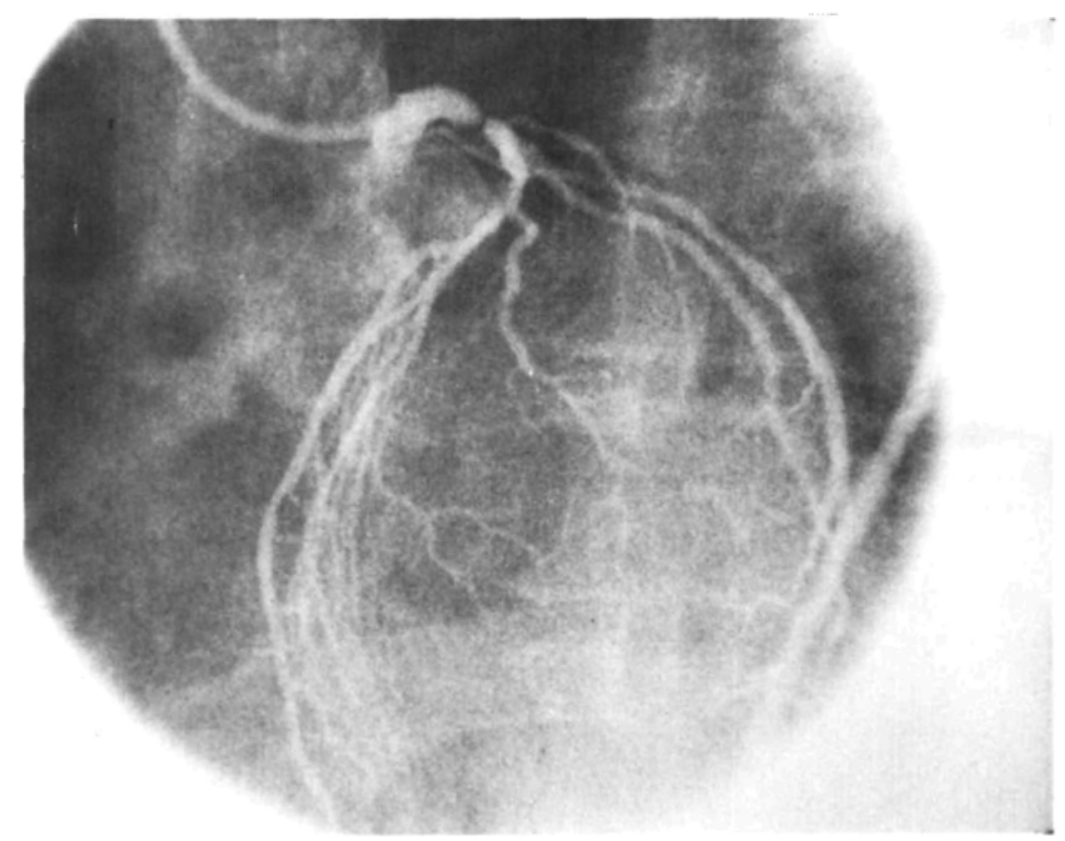

Figune 1. Lésion sténosante sub-totale du tronc commun de l'artère coronaire gauche.

En résumé, les avantages que l'on reconnaît au nitroprussiate seraient les suivants:

(1) Médicament puissant à action prévisible,

(2) action évanescente de courte durée sans besoin d'antidote hypertenseur,

(3) pas d'effet nocif connu sur le débit cardiaque,

(4) pas ou peu de tachyphylaxie,

(5) ne produit de cycloplégie (particulièrement apprécié en neurochirurgie),

(6) médicament bon marché.

\section{Complications de lhypotension contrôlée}

Les complications de cette technique sont bien connues et habituellement sérieuses. Elles sont généralement la conséquence d'ischémie cérébrale, myocardique ou rénale durant l'épisode hypotensif. L'insuffisance coronarienne et cérébrale représentent donc une contre-indication formelle à l'anesthésie sous hypotension. Or comme 20 pour cent de notre population actuellement entre 20 et 60 ans est appelée à mourir de maladie coronarienne, on peut présumer que plusieurs coronariens non diagnostiqués sont susceptibles d'apparaitre parmi nos candidats à l'anesthésie.

L'illustration 1 montre la coronarographie d'un homme de 38 ans porteur d'une lésion unique du tronc commun de la coronaire gauche. Il faut une bonne dose d'audace sinon de franche témérité pour employer l'hypotension contrôlée chez de tels malades.

\section{Indication de lhypotension contrôlée}

L'indication de cette technique se limite à peu près actuellement à ces types d'opérations qui seraient impossibles à réaliser sans elle. Le confort ou le caprice 
du chirurgien ne représente nullement une justification. Si le nitroprussiate de soude arrive à remplir ces promesses, peut-être verrons-nous les indications de l'hypotension contrôlée s'élargir.

En 1956, Little ${ }^{11}$ concluait sa monographie en disant: "L'anesthésie hypotensive porte en elle ses propres complications" et trois ans plus tard, Bodman ${ }^{24}$ corrigeait ce jugement un peu sévère en disant: "Les complications sont attribuables à des erreurs dans l'emploi de la technique." Nous croyons personnellement que cette technique comporte un risque élevé d'erreurs pratiquement inévitables: en hypotension contrôlée, le rapport risque/bénéfice se trouve à la limite inférieure de la tolérabilité.

\section{L'hémodilution}

L'hémodilution comme technique de conservation du sang apparaît beaucoup plus sûre.

Depuis que Francis Moore ${ }^{25}$ en 1959 a recommandé la restriction du sel et de l'eau chez les malades à la suite de chirurgie, les principes de la thérapie liquidienne ont subi plusieurs fluctuations: entre les années 1960 et 1966, plusieurs chercheurs ${ }^{26,27}$ ont rapporté d'importantes réductions du volume liquidien extracellulaire dans les états de chocs et les traumatismes chirurgicaux. Ces auteurs recommandaient l'administration de grandes quantités de solutions électrolytiques équilibrées ( 4 fois la perte sanguine estimée).

Comme 20 pour cent seulement de ce volume infusé demeure intravasculaire, on recommandait en même temps l'administration d'albumine. Par effet osmotique, celle-ci retient dans le compartiment vasculaire trois fois et demi son propre volume. Les portagonistes de la méthode faisaient valoir comme avantages: la stabilité cardiovasculaire, le maintien de la diurèse, la conservation du sang, la diminution de la viscosité et une meilleure perfusion périphérique. Ces auteurs ont montré que l'anémie normovolémique concomitante était bien tolérée.

A la suite de survenue inattendue d'œdème pulmonaire attribuable à cette technique chez des malades chirurgicaux, Moore et Shires ${ }^{28}$ en 1967, s'unirent pour faire un appel à la modération. Pour leur part en 1969, Roth, Lax et Maloney ${ }^{29}$ nièrent l'existence d'un déficit important du liquide extracellulaire chez des chiens soumis à un choc hémorragique. Avant eux, d'autres chercheurs ${ }^{30-32}$ avaient déjà exprimé des doutes sur l'existence ou sur limportance de ce déficit. Cependant, sans vouloir entrer dans la dispute, il senıble que l'hémodilution modérée s'avère physiologiquement faisable et recommandable: la longue expérience de la chirurgie cardiaque avec circulation extracorporelle a confirmé l'innocuité et les bénéfices de l'hémodilution. Cette technique s'est rapidement répandue pour contrer le facteur de viscosité particulièrement important en cours d'hypothermie. Il est aujourd'hui universellement admis que l'hémodilution est bénéfique en chirurgie cardiaque mais on a pas encore démontré de façon probante le niveau idéal d'hématocrite à atteindre. La plupart des centres de chirurgie cardiaque utilisent aujourd'hui un hématocrite entre 15 et 30 pour cent.

Au cours de chirurgies autres que cardiaques, Rush, Richardson, Bossomworth et Eiseman ${ }^{33}$ ont montré en 1969 qe des pertes sanguines entre 400 et $2000 \mathrm{ml}$ pouvaient sans danger être remplacées complètement par les solutions électrolytiques équilibrées. Ce n'est qu'à partir de pertes excédant un litre de sang que 


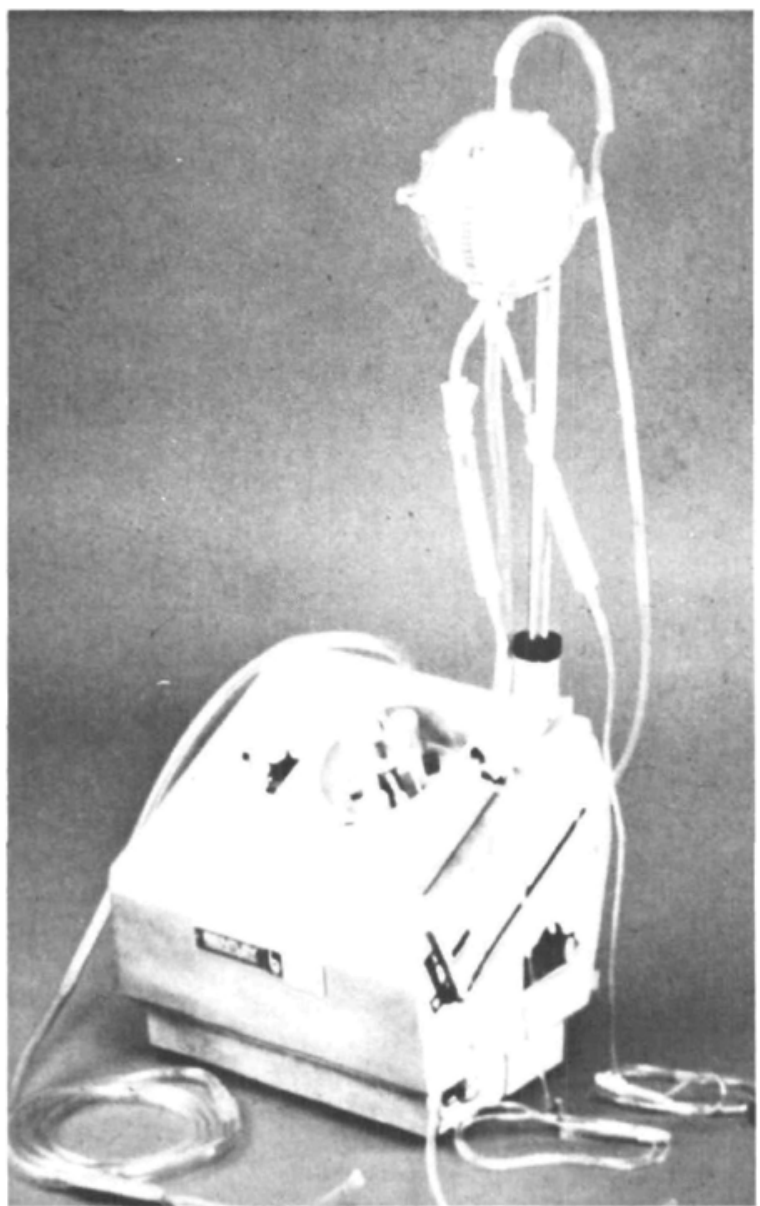

Figure 2, Appareil auto-transfuseur de Bently Laboratories.

des modifications significatives de l'hématocrite, de la protéinémie et du $\mathrm{pH}$ peuvent s'observer.

Tout en reconnaissant que leurs animaux de laboratoire toléraient des hématocrites aussi bas que 8 et 12 pour cent sans signe d'hypoxie tissulaire, ces auteurs ont établi à 28 pour cent d'hématocrite le degré d'hémodilution à utiliser en clinique. Ils avaient démontré dans des travaux antérieurs que le transport d'oxygène aux tissus est encore amplement suffisant à ce degré d'hématocrite. Cette méthode simple et facilement applicable permet, lorsqu'intelligemment employée, d'épargner de grandes quantités de sang sans danger pour les malades.

Transfusions autologues ou Recyclage des pertes sanguines (Intraoperative Blood Salvage)

Cette technique permet de récupérer le sang perdu en cours d'opération et de le réinfuser immédiatement au malade. Cette méthode n'est pas nouvelle: le premier article publié sur ce sujet remonte à 1874 et cléjà en 1931 on trouve 282 publications traitant de son emploi dans des cas d'exsanguination: rupture de grossesse ectopique, anévrisme, blessure de guerre, etc. ... 
Cependant, les découvertes de Landsteiner sur les groupes sanguins et le développement des banques de sang a relégué cette technique dans l'ombre. On manifeste aujourd'hui un intérêt renouvellé pour ce procédé.

\section{PRINCIPES ET MÉTHODES}

Le sang perdu au cours d'une opération, au moment même où il se perd, est recueilli, anticoagulé et retourné à la circulation du malade. En fait, ce recyclage per-opératoire du sang perdu s'emploie régulièrement depuis 1950 en chirurgie cardiaque avec la circulation extracorporelle. C'est à partir de là, que Dyers et ensuite Klebanoff et Watkins conçurent le premier appareil à auto-transfusion qui fut disponible sur le marché. ${ }^{34}$

L'illustration deux montre l'appareil auto-transfuseur de Bentley Laboratories Inc. Cet appareil est essentiellement une pompe qui permet d'aspirer le sang dans un réservoir d'où il peut être retourné directement au malade sous pression ou alors stocké dans des sacs de plastique.

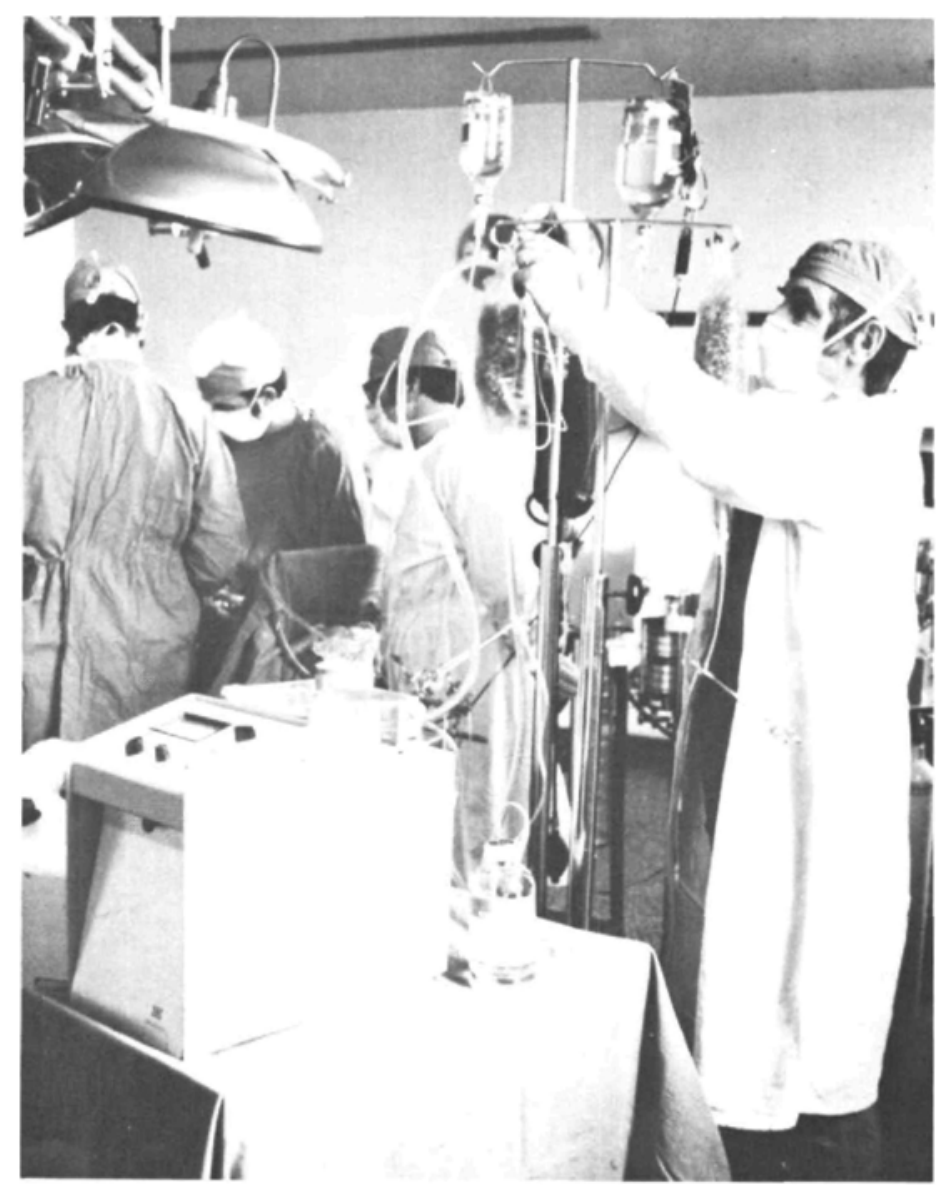

Figure 3. Auto-transfusion: montage-maison. 


\section{Montage maison}

Cherchant à utiliser du matériel déjà disponible dans notre service, nous avons élaboré un montage maison qui nous permet d'effectuer de l'auto-transfusion, sans pouvoir cependant réinfuser immédiatement le sang perdu (illustration 3).

\section{Méthodes}

La solution ACD d'un sac à prélèvements est versée dans la chambre réservoir d'un ensemble à cardiotomie ${ }^{*}$ où le sang recueilli par la succiont s'accumule. Une fois ce réservoir rempli, le sang est transvasé dans un sac de plastique et prêt pour réinfusion au malade. Ce système combine l'hémodilution à l'autotransfusion: en effet, à mesure que l'opération avance, le sang qui se perd est recueilli et immédiatement remplacé généreusement par du lactate Ringer; au moment où nous disposons d'un ou deux flacons de sang à remettre au malade, l'hématocrite est encore bien au-dessus de la limite physiologiquement tolérable.

\section{Précautions}

Un certain degré d'hémolyse est inévitable et il peut être réduit au minimum si l'on prend soin de laisser le sang s'accumuler dans les parties déclives du champ opératoire avant d'être aspiré; il semble préférable que l'aspirateur ramène un flot continu de sang plutôt qu'un mélange d'air et de sang.

Klebanoff dans une étude portant sur 100 patients, rapporte une incidence de 12 pour cent d'hémoglobinémie plasmatique et de 8 pour cent d'hémoglobinurie. ${ }^{35}$ Le phénomène est de courte durée disparaissant en moins de 18 heures et n'a jamais entraîné de complications rénales dans cette série.

Brenner et ses collaborateurs ${ }^{36}$ n'ont pas détecté de complications rénales chez des malades auto-transfusés ayant des concentrations d'hémoglobine plasmatique de 50 à $600 \mathrm{mg}$ par $100 \mathrm{ml}$. Il souligne qu'il est très important avec ces techniques de maintenir une hydratation généreuse, un diurèse osmotique et d'éviter toute hypotension ou acidose.

\section{Indications}

Cette technique est applicable dans les cas où l'on prévoit une perte de sang importante. On hésite généralement à l'employer dans la chirurgie pour tumeur maligne et pour des cas infectés.

Notre expérience avec cette technique est encore limitée, mais elle est suffisamment étendue pour nous permettre d'affirmer que la technique est simple, facile à maîtriser et très efficace pour conserver le sang.

\section{ConClusion}

On peut dire que la médecine a longtemps eu à l'égard de la transfusion sanguine la même attitude que notre société en général à l'égard du pétrole: comme le pétrole, on a considéré le sang comme une ressource inépuisable et sans danger. Or comme celui-ci, le sang coûte plus cher, il est en passe de devenir plus rare,

•Cardiotomy Reservoir (Baxter 5 M 0386).

fTravenol Laboratories Model 3500 Portable Pump. 
et comme lui, il risque occasionnellement de modifier désavantageusement l'écologie intérieure du malade.

Ce travail a voulu attirer l'attention sur ce sujet.

\section{RÉSUMÉ}

Divers facteurs contribuent déjà à limiter la quantité de sang de banque disponible et il faut peut-être même prévoir une réduction des arrivages devant la désaffection relative de la jelnesse à l'égard du don de sang et la multiplication des porteurs d'antigène Australien.

De plus, de nouvelles opérations surgissent, qui augmentent les besoins. Il est impérieux d'adopter des attitudes et pratiquer des techniques visant à conserver le sang.

Dans cette optique, ce travail présente les avantages d'un comité d'étude des transfusions qui, à lui seul, peut réduire la consommation de sang de 20 pour cent; de plus, on passe en revue les techniques proprement dites visant à épargner du sang, soit: l'hypotension contrôlée en anesthésie chirurgicale, l'hémodilution et l'auto-transfusion.

\section{SUMMARY}

Many factors already contribute to limit the amount of bank blood available for therapeutic use; a possible reduction in the amount of available blood could stem from the relative indifference of young people towards the gift of blood and the increase in the size of the pool of positive carriers of Australian antigen.

Moreover, new operations appear (coronary by-pass) which increase the demand for blood.

It is imperative that we adopt attitudes and practice techniques that will contribute to the conservation of blood.

This paper discusses the advantages of an active blood bank committee which, alone, can reduce the consumption of blood by 20 per cent.

Moreover, actual techniques for conservation of blood are reviewed: controlled hypotension, haemodilution and intraoperative blood salvage.

\section{BIBLIOGRAPHIE}

1. Pefreault, R.A. Directeur National Service de Transfusion de la Croix Rouge. Communication personnelle (1974).

2. Conference Report: Serum hepatitis: a major challenge to workers in infectious and iatrogenic disease. Canad. Med. Assoc. J. 110: 974 (1974).

3. Roche, J.K. \& STEngle, J.M. Open-heart surgery and the demand for blood. J.A.M.A. 225: 1515 (1973).

4. Lawson, N.W., Ochner, J.L., Mills, N.L., \& LÉonahd, G.L. The use of hemodilution and fresh autologous blood in open-heart surgery. Anesth. \& Analg. 53: 672 (1974).

5. Walz, D.V. An effective hospital transfusion committee. J.A.M.A. 189: 660 (1964).

6. Allen, G.J. The case for the single transfusion. Editorial. New Engl. J. Med. 287: 984 (1972).

7. ReEce, R.L. \& BecketT, R.S. Epidemiology of single unit transfusion. J.A.M.A. 195: 113 (1966).

8. Okuno, T. The Cunningham case and blood usage. Letter to Editor. J.A.M.A. 220: 1015 (1972). 
9. Devitt, J.E. Blood wastage and cholecystectomy. Canad. Med. Assoc. J. 109: 120 (1973).

10. Shier, K.J. Letter to Editor: Blood wastage. Canad. Med. Assoc. J. 109: 1078 (1973).

11. Little, D.M., JR. Controlled hypotension in anesthesia and surgery. Charles C. Thomas, Illinois (1956).

12. Eckenoff, J.E. Ann. N.Y. Acad. Sciences 115: 341 (1964).

13. Wylie, W.D. \& Chunchil.L-Davidson, H.C. A practice of anaesthesia. 2ième édit. Chicago, Year Medical Publisher.

14. Jorwan, W.S., Graves, C.L., Boyd, W.A., Ueda, I., \& Roberts, T.S.S. Cardiovascular effects of three technics for inducing hypotension during anesthesia. Anesth. \& Analg. 50: 1059 (1971).

15. Jones, G.O.M. \& Cole, P. Sodium nitroprusside as a hypotensive agent. Brit. J. Anaesth. 40: 804 (1968).

16. Schiffmann, H. \& Fuchs, P. Controlled hypotension effected by sodium nitroprusside. Acta. Anesth. Scand., Suppl, 23: 704 (1966).

17. Moraca, P.P., Brtte, E.M., Hale, D.E., Wasmuth, O.E., \& Poutasse, E.F. Clinical evaluation of sodium nitroprusside as a hypotensive agent. Anesthesiology 23: 193 (1962).

18. Wildsmith, J.A.W., Marshall, R.L., Jenkinson, J.L., Macrae, W.R., \& Scott, D.B. Hemodynamic effects of sodium nitroprusside during nitrous oxide/halothane anaesthesia, Brit. J. Anaesth. 45: 71 (1973).

19. Styles, M., Coleman, A.J., \& Leary, W.P. Some hemodynamic effects of sodium nitroprusside. Anesthesiology 38: 173 (1973).

20. Boxer, G.E. \& Rickards, J.C. Studies on the metabolism of the carbon cyanide and thiocyanate. Arch. Biochem. 39: 7 (1952).

21. Goldstern, F. \& Riedens, F. Conversion of thiocyanate to cyanide by an etrythropoietic enzyme. Amer. J. Physiol. 173: 287 ( 1953).

22. Schlant, R.C., Tsacarts, T.S., \& Robertson, R.J. Studies on the acute cardiovascular effects of intravenous sodium nitroprusside. Anesthesiology 38: 51 (1973).

23. Franciosa, J.A., Guina, N.H., Lmas, D.J., et al. Improved left ventricular function during nitroprusside infusion in acute myocardial infarction. Lancet 1: 650 (1972).

24. Bodman, R.I. Controlled hypotension. Internation. Anaesthesiology Clinics 5: 90 (1967).

25. Moone, F.D. Metabolic care of surgical patient. Philadelphia and London, W.B. Saunders Company (1959).

26. ВовА, A. Support of blood volume during operation without blood transfusion. Surg. Forum 17: 61 (1966).

27. Shires, T., Williams, J., \& Brown, F. Acute change in extracellular fluids associated with major surgical procedures. Ann. Surg. 154: 803 (1961).

28. Moone, F.D. \& Shires, G.T. Moderation. Editorial. Ann. Surg. 166: 300 (1967).

29. Roth, E., Lax, L.C., \& Maloney, J.V. Ringer's lactate solution and extracellular fluid volume in the surgical patient: A critical analysis. Ann. Surg. 169: 149 (1969).

30. Serkes, K.D. \& Lanc, S. Changes in extracellular fluid volume after hemorrage and tournique trauma. Surg. Forum 17: 58 (1966).

31. Virtue, R.W., Levine, D.S., \& Amawa, J.K. Fluid shifts during the surgical period RISA and $S$ determination following glucose, saline or lactate infusion. Ann. Surg. 163: 523 (1966).

32. Cleland, J., Pluth, J.R., Tauxe, W.N., \& Kirklin, J.W. Blood volume and body fluid compartment changes soon after closed and open intracardiac surgery. J. Thorac. Cardiovasc. Surg. 52: 698 (1966)

33. Rush, B.F., Richardson, J.D., Bosomworth, P., \& Eiseman, B. Limitations of blood replacement with electrolyte solutions. Arch. Surg. 98: 49 (1969).

34. Wilson, J.D. \& TAswel. , H.F. Autotransfusion: Historical review and preliminary report on a new method. Mayo Clin. Proc. 43: 26 (1968).

35. Brenner, B.J., Raines, J.K., \& Darling, R.C. Intra-operative auto-transfusion in abdominal aortic resections. Arch. Surg. 107: 78 (1973).

36. Klebanoff, G. An experience of 100 cases of introperative auto-transfusion with a commercially available device. Read before the 25th meeting of the American Association of Blood Banks. Washington DC (1972). 